\title{
Finite Deformation Guided Nonlinear Filtering for Multiframe Cardiac Motion Analysis
}

\author{
C.L. Ken Wong and Pengcheng Shi \\ Biomedical Research Laboratory \\ Department of Electrical and Electronic Engineering \\ Hong Kong University of Science and Technology \\ Clear Water Bay, Kowloon, Hong Kong \\ \{eewclken, eeship\}@ust.hk
}

\begin{abstract}
In order to obtain sensible estimates of myocardial kinematics based on biomechanics constraints, one must adopt appropriate material, deformation, and temporal models. Earlier efforts, although not concurrently adopted within the same framework, have shown that it is essential to carefully consider the fibrous structure of the myocardium, the large geometric deformation of the cardiac wall movement, the multiframe observations over the cardiac cycle, and the uncertainties in the system modeling and data measurements. With the meshfree particle method providing the platform to enforce the anisotropic material property derived from the myofiber architecture, we present the first effort to perform multiframe cardiac motion analysis under finite deformation conditions, posed as a nonlinear statistical filtering process. Total Lagrangian (TL) formulation is adopted to establish the myocardial system dynamics under finite deformation, which is then used to perform nonlinear state space prediction (of the tissue displacement and velocity) at each time frame, using the Newton-Raphson iteration scheme. The system matrices of the state space equation are then derived, and the optimal estimation of the kinematic state is achieved through TL-updated recursive filtering. Results from synthetic data with ground truth and canine cardiac image sequence are presented.
\end{abstract}

\section{Introduction}

In cardiac motion analysis, the imaging data typically only provide noisy measurements at some salient landmark points, such as the Lagrangian tag displacements from the MR tagging images and the Eulerian tissue velocities from the MR phase contrast data. In order to obtain the complete cardiac motion field, a priori material, deformation, and temporal constraints are required to obtain a unique solution in some optimal sense 3].

For successful and meaningful cardiac motion recovery using biomechanical models, the myocardial constitutive laws and the deformation properties need to be properly considered [4. From experimental testings, it has been shown that the material properties along and cross the myofibers are substantially 
different. Furthermore, it has long been observed that normal cardiac deformation (radial contraction) reaches at least 30\% between end-diastole (ED) and end-systole (ES). Hence, spatial constraints with anisotropic properties and finite deformation models are required for realistic cardiac motion analysis. Sofar, however, with few recent exceptions [511, most of the efforts have been using the isotropic material model for computational simplicity [3]. Moreover, except our most recent work [12, no algorithm has undergone the essential finite deformation analysis. In addition, multiframe analysis is also of paramount importance for cardiac motion recovery. The temporal kinematics coherence plays key roles in achieving robust motion estimates, especially when there are uncertainties in system models and noises in input data $7 / 8 \mid 10$. Unfortunately, none of these multiframe works have employed the proper anisotropic and finite deformation constraints.

In our recent work, the importance of using anisotropic material and finite deformation models has been demonstrated for frame-to-frame analysis 12 . We have also shown the advantages of using the meshfree particle method (MPM) to deal with myofiber orientations, geometric and kinematics discontinuities, and representation refinements. In this paper, we extend that effort to perform multiframe analysis under finite deformation conditions as a nonlinear statistical filtering problem. Total Lagrangian (TL) formulation is adopted to establish the myocardial system dynamics under large deformation, which then relies on the Newton-Raphson scheme to perform nonlinear predictions of the displacement and velocity for the next time frame. The system matrices of the state space representation are then derived, and the optimal estimation of the kinematic state is achieved through TL-updated recursive filtering. Simulations on synthetic data have shown superior performance over existing strategies, and experiments with canine MR images have produced physiologically sensible outcomes.

\section{Methodology}

\subsection{Meshfree Particle Representation of Myocardium}

Using the meshfree particle method, the myocardium can be represented by a set of unstructured, adaptively sampled nodes, bounded by the segmented endo- and epi-cardial boundaries (see Fig. 2 for a MPM represented 2D LV slice). Let $u(\mathbf{x})$ be the displacement field of the myocardial tissue at point $\mathbf{x}$, the approximated displacement function $u^{h}(\mathbf{x})$ is then given by the moving least square (MLS) approximation: $u^{h}(\mathbf{x})=\sum_{I=1}^{N} \phi_{I}(\mathbf{x}) u_{I}$, where $\phi_{I}(\mathbf{x})$ is the MLS shape function of node $I, N$ is the total number of sampling nodes, and $u_{I}$ is the nodal displacement value [8].

\subsection{Anisotropic Composite Material Model}

Realistic anisotropic material models are essential for the accurate recovery of cardiac movement. For elastic materials, both isotropic and anisotropic, the 2D stress-strain relationships obey the Hooke's Law: 


$$
\left[\begin{array}{l}
S_{11} \\
S_{22} \\
S_{12}
\end{array}\right]=C\left[\begin{array}{l}
\epsilon_{11} \\
\epsilon_{22} \\
\epsilon_{12}
\end{array}\right]
$$

where $\epsilon_{i j}$ are the components of the Green-Lagrangian strain tensor, $S_{i j}$ are the components of the second Piola-Kirchhoff (PKII) stress tensor, and $C$ is the stiffness matrix. Let the $2 \mathrm{D}$ stiffness matrix of a point with $0^{\circ}$ fiber orientation be $C_{o}$ and of the form:

$$
C_{o}=\left[\begin{array}{ccc}
1 / E_{f} & -\nu / E_{f} & 0 \\
-\nu / E_{f} & 1 / E_{c f} & 0 \\
0 & 0 & 1 / G
\end{array}\right]^{-1}
$$

where $E_{c f}$ and $E_{f}$ are the cross-fiber and along-fiber Young's modulus respectively, $\nu$ is the Poisson ratio measuring the material compressibility, and $G \approx E_{f} /(2(1+v))$ describes the shearing properties. Then, the stiffness matrix at any point with fiber orientation $\theta$ can be calculated from $C_{o}$ through $C_{\theta}=T^{-1} C_{o} R T R^{-1}[9]$, where $T$ is the coordinate transformation matrix which is a function of $\theta$, and $R$ is a matrix responsible for the transformation between the strain tensor components and the engineering strain tensor components.

\subsection{Myocardial System Dynamics under Finite Deformation}

When the deformation is large, the strain calculated by directly using the linearized Green-Lagrangian strain calculation would not be accurate because the term $\frac{1}{2} u_{i, k} u_{j, k}$ in the full Green-Lagrangian strain calculation $\epsilon_{i j}=\frac{1}{2}\left(u_{i, j}+\right.$ $u_{j, i}+u_{k, i} u_{k, j}$ ) (where $u_{i, j}=\partial u_{i} / \partial x_{j}$ ) becomes large. Hence, to proper treat all kinematics nonlinear effects caused by large rotations, large displacements, and large strains, the dynamic equilibrium equation with linearized incremental Total Lagrangian (TL) formulation should be used [1].

Using the displacement field approximation derived at Section 2.1, the TL represents the equilibrium at time $t+\Delta t$ in the following form 1$]$ [1]:

$$
\left({ }_{0}^{t} \mathbf{K}_{L}+{ }_{0}^{t} \mathbf{K}_{N L}\right) \Delta \mathbf{U}={ }_{0}^{t} \tilde{\mathbf{K}} \Delta \mathbf{U}={ }^{t+\Delta t} \mathbf{R}-{ }_{0}^{t} \mathbf{F}
$$

where ${ }_{0}^{t} \mathbf{K}_{L}$ and ${ }_{0}^{t} \mathbf{K}_{N L}$ are the linear and nonlinear strain incremental stiffness matrices, respectively, ${ }^{t+\Delta t} \mathbf{R}$ is the external force, ${ }_{0}^{t} \mathbf{F}$ is the force related to the stress, and $\Delta \mathbf{U}=\left[\Delta u_{1}, \Delta u_{2}, \ldots . . \Delta u_{N}\right]^{T}$ is the incremental displacement vector caused by the force difference ${ }^{t+\Delta t} \mathbf{R}-{ }_{0}^{t} \mathbf{F}$.

Equation (3) is the complete governing equation for static finite deformation analysis. By considering the inertia of the system, it becomes the system governing equation for dynamic finite deformation analysis [1]:

$$
{ }_{0}^{t} \mathbf{M}{ }^{t+\Delta t} \ddot{\mathbf{U}}+{ }_{0}^{t} \mathbf{C}{ }^{t+\Delta t} \dot{\mathbf{U}}+{ }_{0}^{t} \tilde{\mathbf{K}} \Delta \mathbf{U}={ }^{t+\Delta t} \mathbf{R}-{ }_{0}^{t} \mathbf{F}
$$

where ${ }^{t+\Delta t} \ddot{\mathbf{U}}$ and ${ }^{t+\Delta t} \dot{\mathbf{U}}$ are the acceleration and velocity at time $t+\Delta t$ respectively, ${ }_{0}^{t} \mathbf{M}$ is the mass matrix, and ${ }_{0}^{t} \mathbf{C}$ is the damping matrix.

\footnotetext{
${ }^{1}$ In all equations, the left subscript indicates the time at which the measurement is referred to, and the left superscript indicates the time at which the quantity is measured.
} 


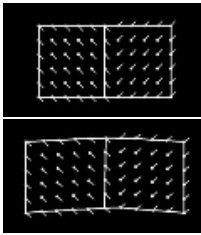

(a)

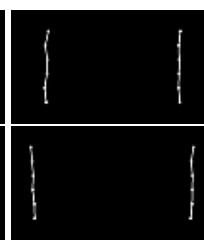

(b)

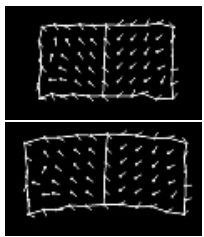

(c)

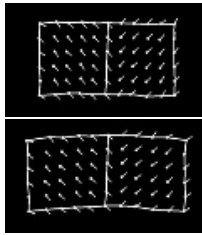

(d)

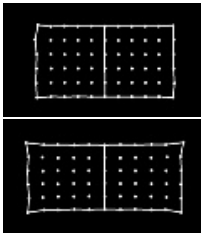

(e)

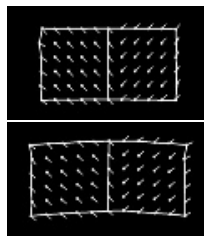

(f)

Fig. 1. Experiments on the synthetic data. (a): Two frames (\#1 and \#9, out of sixteen) of a deforming object which is composed of two materials. Generated by enforcing outward boundary displacements on the left and right edges, with frame \#1 the original configuration. (b): Noisy displacement observations on the left and right edges $(\mathrm{SNR}=2.919 d B)$, used as data inputs. $(\mathrm{c})-(\mathrm{f})$ : Recovered object geometry from the noisy data, using: frame-to-frame estimation with anisotropic material and finite deformation models (c); multiframe estimation with anisotropic material and infinitesimal deformation models (d); multiframe estimation with isotropic material and finite deformation models (e); and multiframe estimation with anisotropic material and finite deformation models (f).

\subsection{Frame-to-Frame Nonlinear Kinematics Analysis}

While solving for Equation (4), in order to reduce the error introduced by the linearization at any particular time instance, the Newton-Raphson iteration is employed [1. Using the trapezoidal rules, i.e. ${ }^{t+\Delta t} \dot{\mathbf{U}}={ }^{t} \dot{\mathbf{U}}+\frac{\Delta t}{2}\left({ }^{t} \ddot{\mathbf{U}}+{ }^{t+\Delta t} \ddot{\mathbf{U}}\right)$ and ${ }^{t+\Delta t} \mathbf{U}={ }^{t} \mathbf{U}+\frac{\Delta t}{2}\left({ }^{t} \dot{\mathbf{U}}+{ }^{t+\Delta t} \dot{\mathbf{U}}\right)$, and the displacement update equation during the Newton-Raphson iteration ${ }^{t+\Delta t} \mathbf{U}^{k}={ }^{t+\Delta t} \mathbf{U}^{k-1}+\Delta \mathbf{U}^{k}$ where the right superscript $k$ indicates the iteration and ${ }^{t+\Delta t} \mathbf{U}^{0}={ }^{t} \mathbf{U}$, then we have

$$
\begin{aligned}
{ }^{t+\Delta t} \dot{\mathbf{U}}^{k} & =\frac{2}{\Delta t}\left({ }^{t+\Delta t} \mathbf{U}^{k-1}+\Delta \mathbf{U}^{k}-{ }^{t} \mathbf{U}\right)-{ }^{t} \dot{\mathbf{U}} \\
{ }^{t+\Delta t} \ddot{\mathbf{U}}^{k} & =\frac{4}{\Delta t^{2}}\left({ }^{t+\Delta t} \mathbf{U}^{k-1}+\Delta \mathbf{U}^{k}-{ }^{t} \mathbf{U}\right)-\frac{4}{\Delta t}{ }^{t} \dot{\mathbf{U}}-{ }^{t} \ddot{\mathbf{U}}
\end{aligned}
$$

Substituting Equations (5) and (6) into Equation (4), after rearrangement, we obtain the governing iteration equation between time $t$ and time $t+\Delta t$ as 2 :

$$
\hat{\mathbf{K}}^{k-1} \Delta \mathbf{U}^{k}=\Delta \mathbf{R}^{k-1}-\left(\hat{\mathbf{K}}^{k-1}-\tilde{\mathbf{K}}^{k-1}\right) \mathbf{U}^{k-1}
$$

where

$$
\begin{aligned}
\hat{\mathbf{K}}^{k-1} & =\frac{4}{\Delta t^{2}} \mathbf{M}^{k-1}+\frac{2}{\Delta t} \mathbf{C}^{k-1}+\tilde{\mathbf{K}}^{k-1} \\
\Delta \mathbf{R}^{k-1} & ={ }^{t+\Delta t} \mathbf{R}-\mathbf{F}^{k-1} \\
& -\mathbf{M}^{k-1}\left(-\frac{4}{\Delta t^{2}}{ }^{t} \mathbf{U}-\frac{4}{\Delta t}{ }^{t} \dot{\mathbf{U}}-{ }^{t} \ddot{\mathbf{U}}\right)-\mathbf{C}^{k-1}\left(-\frac{2}{\Delta t}{ }^{t} \mathbf{U}-{ }^{t} \dot{\mathbf{U}}\right)
\end{aligned}
$$

\footnotetext{
${ }^{2}$ In order to keep the equation simple to read, we assume that any parameter with right superscript $k$ is measured at time $t+\Delta t$, thus we can omit the left scripts indicating the time.
} 
Table 1. Positional differences between the ground truth and estimated nodal locations under different models.

\begin{tabular}{|c|c|c|c|c|}
\hline $\begin{array}{c}\text { temporal } \\
\text { material } \\
\text { deformation }\end{array}$ & $\begin{array}{c}\text { frame-to-frame } \\
\text { anisotropic } \\
\text { finite }\end{array}$ & $\begin{array}{c}\text { multi-frame } \\
\text { anisotropic } \\
\text { infinitesimal }\end{array}$ & $\begin{array}{c}\text { multi-frame } \\
\text { isotropic } \\
\text { finite }\end{array}$ & $\begin{array}{c}\text { multi-frame } \\
\text { anisotropic } \\
\text { finite }\end{array}$ \\
\hline Frame \#1 & $0.31170 \pm 0.25571$ & $0.18633 \pm 0.09015$ & $0.15672 \pm 0.12818$ & $0.10805 \pm 0.10021$ \\
Frame \#9 & $0.33567 \pm 0.20786$ & $0.21138 \pm 0.12648$ & $0.32348 \pm 0.19121$ & $0.10881 \pm 0.10293$ \\
\hline
\end{tabular}

with $\mathbf{M}^{0}={ }_{0}^{t} \mathbf{M}, \mathbf{C}^{0}={ }_{0}^{t} \mathbf{C}, \mathbf{K}^{0}={ }_{0}^{t} \mathbf{K}$, and $\mathbf{F}^{0}={ }_{0}^{t} \mathbf{F}$. The governing iteration equation (7) would iterate until the unbalanced dynamic force $\Delta \mathbf{R}^{k-1}-\left(\hat{\mathbf{K}}^{k-1}-\right.$ $\left.\tilde{\mathbf{K}}^{k-1}\right) \mathbf{U}^{k-1}$ approaches zero.

Using Equation (7), the displacement update equation during the NewtonRaphson iteration becomes

$$
\mathbf{U}^{k}=\left(\hat{\mathbf{K}}^{k-1}\right)^{-1} \tilde{\mathbf{K}}^{k-1} \mathbf{U}^{k-1}+\left(\hat{\mathbf{K}}^{k-1}\right)^{-1} \Delta \mathbf{R}^{k-1}
$$

Let $\bar{k}$ be the iteration at which convergence occurs, and let $A^{k-1}=$ $\left(\hat{\mathbf{K}}^{k-1}\right)^{-1} \tilde{\mathbf{K}}^{k-1}$ and $B^{k-1}=\left(\hat{\mathbf{K}}^{k-1}\right)^{-1} \Delta \mathbf{R}^{k-1}$. With Equation (8), the relation between $\mathbf{U}^{\bar{k}}$ and $\mathbf{U}^{0}$ is derived to be

$$
\mathbf{U}^{\bar{k}}=\left(\prod_{i=0}^{\bar{k}-1} A^{i}\right) \mathbf{U}^{0}+\sum_{i=1}^{\bar{k}-1}\left(\left(\prod_{j=i}^{\bar{k}-1} A^{j}\right) B^{i-1}\right)+B^{\bar{k}-1}
$$

Using this equation, Equation (5) can be written into

$$
\dot{\mathbf{U}}^{\bar{k}}=\frac{2}{\Delta t}\left(\prod_{i=0}^{\bar{k}-1} A^{i}-\mathbf{I}\right) \mathbf{U}^{0}-\dot{\mathbf{U}}^{0}+\frac{2}{\Delta t}\left(\sum_{i=1}^{\bar{k}-1}\left(\left(\prod_{j=i}^{\bar{k}-1} A^{j}\right) B^{i-1}\right)+B^{\bar{k}-1}\right)
$$

\subsection{State Space Analysis of Nonlinear System Dynamics: Optimal Multiframe Kinematics Estimation}

Since $\mathbf{U}^{\bar{k}}={ }^{t+\Delta t} \mathbf{U}, \mathbf{U}^{0}={ }^{t} \mathbf{U}, \dot{\mathbf{U}}^{\bar{k}}={ }^{t+\Delta t} \dot{\mathbf{U}}$, and $\dot{\mathbf{U}}^{0}={ }^{t} \dot{\mathbf{U}}$, Equations (9) and (10) can be combined in the form

$$
x(t+\Delta t)=D(t+\Delta t) x(t)+w(t)
$$

where

$$
\begin{aligned}
D(t+\Delta t) & =\left[\begin{array}{cc}
\prod_{i=0}^{\bar{k}-1} A^{i} & 0 \\
\frac{2}{\Delta t}\left(\prod_{i=0}^{\bar{k}-1} A^{i}-\mathbf{I}\right) & -\mathbf{I}
\end{array}\right], \\
w(t) & =\left[\begin{array}{c}
\sum_{i=1}^{\bar{k}-1}\left(\left(\prod_{j=i}^{\bar{k}-1} A^{j}\right) B^{i-1}\right)+B^{\bar{k}-1} \\
\frac{2}{\Delta t}\left(\sum_{i=1}^{\bar{k}-1}\left(\left(\prod_{j=i}^{\bar{k}-1} A^{j}\right) B^{i-1}\right)+B^{\bar{k}-1}\right)
\end{array}\right], \\
x(t+\Delta t) & =\left[\begin{array}{c}
{ }^{t+\Delta t} \mathbf{U} \\
t+\Delta t \\
{ }^{t+} \dot{\mathbf{U}}
\end{array}\right], \text { and } x(t)=\left[\begin{array}{c}
{ }^{t} \mathbf{U} \\
{ }^{t} \dot{\mathbf{U}}
\end{array}\right] .
\end{aligned}
$$



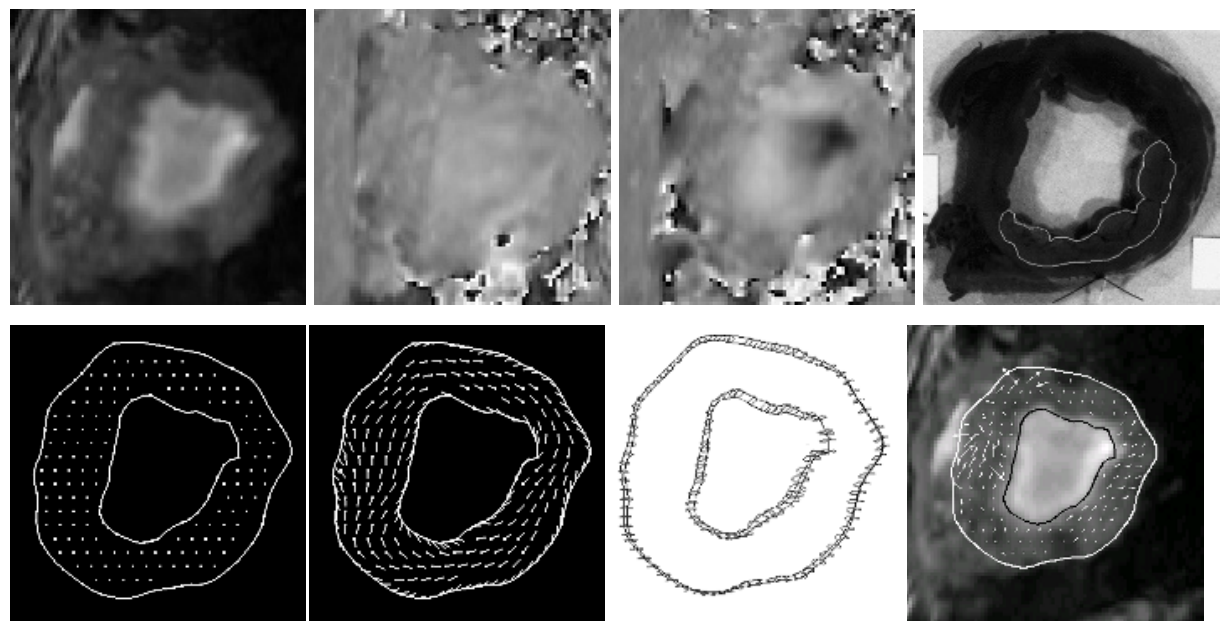

Fig. 2. Canine MRI phase contrast data. From left to right: MR intensity, x-velocity, yvelocity, and TTC-stained post mortem myocardium with infarcted tissue highlighted (top); meshfree representation of the slice, myofiber orientations, boundary displacement constraints, and phase contrast velocity field (bottom).

Including the zero-mean, additive, and white process noise $v(t)(E[v(t)]=0$, $\left.E\left[v(t) v(s)^{\prime}\right]=Q_{v}(t) \delta_{t s}\right)$, Equation (11]) becomes the TL-updated state-space equation which has performed nonlinear state prediction of tissue displacement and velocity using the Newton-Raphson iteration scheme:

$$
x(t+\Delta t)=D(t+\Delta t) x(t)+w(t)+v(t)
$$

Since the data observations $y(t+\Delta t)$ obtained from the imaging data are corrupted by noise $e(t)$ (once again, assuming zero-mean, additive, and white, with $\left.E[e(t)]=0, E\left[e(t) e(s)^{\prime}\right]=R_{e}(t) \delta_{t s}\right)$, the measurement equation thus is:

$$
y(t+\Delta t)=H x(t+\Delta t)+e(t+\Delta t),
$$

where $H$ is a known, user-specified measurement matrix.

With the discretized state space equations (12) and (13), the multiframe estimation of the cardiac kinematics over the cardiac cycle can be performed using standard recursive filtering procedures until convergence [6]:

1. Initialize state and error covariance estimates, $\hat{x}(t)$ and $P(t)$.

2. Prediction step: using the filter update equations to predict the state and error covariance at time $t+\Delta t$ :

$$
\begin{aligned}
\hat{x}^{-}(t+\Delta t) & =D(t+\Delta t) \hat{x}(t)+w(t) \\
P^{-}(t+\Delta t) & =D(t+\Delta t) P(t) D^{T}(t+\Delta t)+Q_{v}(t)
\end{aligned}
$$



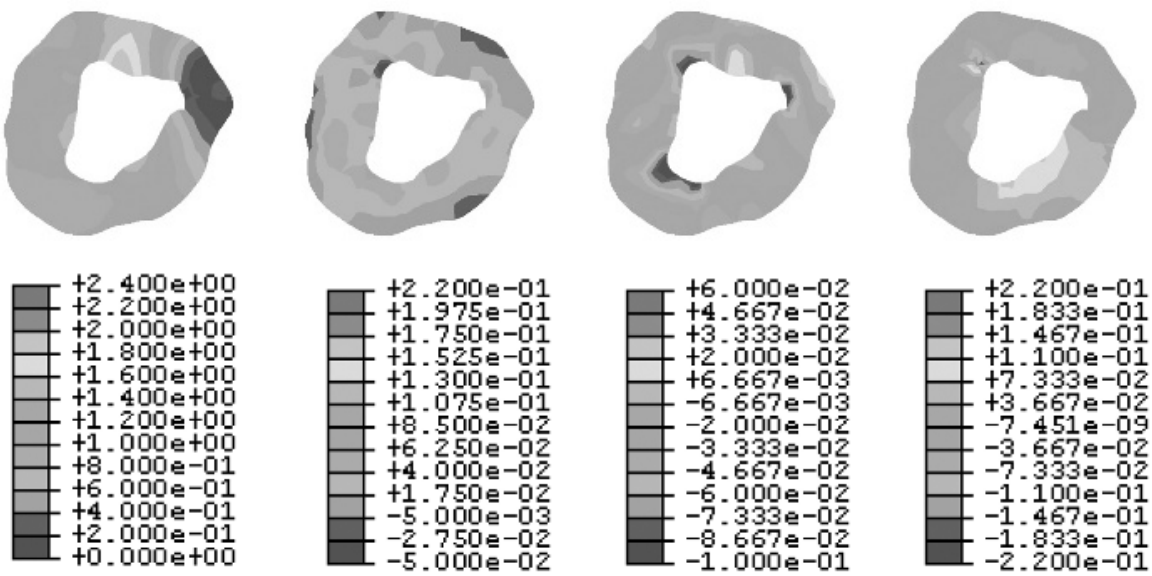

Fig. 3. Estimated displacement, and radial, circumferential, and RC shear strain maps for frame \#9 (with respective to frame \#1).

3. Correction step: with the use of filter gain $G(t+\Delta t)$, the predictions are updated using the measurements $y(t+\Delta t)$ :

$$
\begin{aligned}
G(t+\Delta t) & =P^{-}(t+\Delta t) H^{T}\left(H P^{-}(t+\Delta t) H^{T}+R_{e}(t+\Delta t)\right)^{-1} \\
\hat{x}(t+\Delta t) & =\hat{x}^{-}(t+\Delta t)+G(t+\Delta t)\left(y(t+\Delta t)-H \hat{x}^{-}(t+\Delta t)\right) \\
P(t+\Delta t) & =(I-G(t+\Delta t) H) P^{-}(t+\Delta t)
\end{aligned}
$$

\section{$3 \quad$ Experiments}

\subsection{Synthetic Data}

As shown in Fig. 1, experiments have been conducted on a synthetic image sequence (16 frames) with different material, deformation, and temporal models to verify the needs to adopt the proper model constraints. The importance of using multiframe filtering can be observed by comparing Fig. 1(c) to Fig. 1(d,e,f). From Fig. 1(d) and (f), it can be seen that Fig. [1(d) deviates comparatively more than Fig. 1(f). This is because when deformation is large, the error caused by linearized strain tensor would accumulate during the filtering loop, so the object gradually deforms away from the configuration at the previous loop and would not converge. The importance of using anisotropic material model is shown between Fig. 1(e) and (f). Similarly, it can be seen from Table. 1 that the deformed geometry using multiframe estimation with finite deformation and anisotropic material models deviates the least from the ground truth. 


\subsection{In Vivo Canine MRI Data}

Experiments have also been performed using a canine cardiac phase contrast MRI sequence (Fig 21), which contains the cardiac anatomy and also the respective instantaneous velocities of the tissues. The myofiber orientations are mapped onto the particular image slice, based on the principal warps algorithm of the landmarks [2]. The heart boundaries and their shape-based displacements over the 16-frame cardiac cycle are estimated [13], along with the mid-wall phase contrast velocities (Fig 2). The recovered displacement and cardiac-specific strain maps are shown in Fig. 3 Compared to our earlier, frame-to-frame results [12, these outcomes are overall better correlated with the TTC stained tissue.

This work is supported, in part, by HKRGC CERG Grant HKUST6151/03E.

\section{References}

1. K.J. Bathe. Finite Element Procedures. Prentice Hall, 1996.

2. F.L. Bookstein. Principal warps: Thin-plate splines and the decomposition of deformations. IEEE PAMI, 11:567-585, 1989.

3. A.J. Frangi, W.J. Niessen, and M.A. Viergever. Three-dimensional modeling for functional analysis of cardiac images: A review. IEEE TMI, 20:2-25, 2001.

4. L. Glass, P. Hunter, and A. McCulloch, editors. Theory of Heart. Springer, 1991.

5. Z. Hu, D. Metaxas, and L. Axel. In vivo strain and stress estimation of the heart left and right ventricles from MRI images. Medical Image Analysis, 7:435-444, 2003.

6. E.W. Kamen and J.K. Su. Introduction to Optimal Estimation. Springer, 1999.

7. W.S. Kerwin and J.L. Prince. The kriging update model and recursive space-time function estimation. IEEE TSP, 47:2942-2952, 1999.

8. H. Liu and P. Shi. Meshfree representation and computation: applications to cardiac motion analysis. In IPMI'03, pages 560-572, 2003.

9. F.L. Matthews and R.D. Rawlings. Composite Materials. Chapman \& Hall, 1994.

10. J.C. McEachen and J.S. Duncan. Multiframe temporal estimation of cardiac nonrigid motion. IEEE TMI, 16:270-283, 1997.

11. X. Papademetris, A.J. Sinusas, D.P. Dione, R.T. Constable, and J.S. Duncan. Estimation of $3 \mathrm{D}$ left ventricular deformation from medical images using biomechanical models. IEEE TMI, 21:786-799, 2002.

12. C.L. Wong, H. Liu, L.N. Wong, A.J. Sinusas, and P. Shi. Meshfree cardiac motion analysis framework using composite material model and total Lagrangian formulation. In IEEE ISBI'04, pages 464-467, 2004.

13. L.N. Wong, H. Liu, A. Sinusas, and P. Shi. Spatio-temporal active region model for simultaneous segmentation and motion estimation of the whole heart. In IEEE VLSM'03, pages 193-200, 2003. 